\title{
ON A CONTINUED FRACTION OF ORDER 12
}

\author{
K. R. Vasuki, Abdulrawf A.A. Kahtan, G. Sharath, and C. Sathish Kumar
}

UDC 511.72

We present some new relations between a continued fraction $U(q)$ of order 12 (established by M. S. M. Naika et al.) and $U\left(q^{n}\right)$ for $n=7,9,11,13$.

\section{Introduction}

Throughout the paper, we assume that $|q|<1$. For a positive integer $n$, we use the following standard notation:

$$
\begin{gathered}
(a)_{0}:=(a ; q)_{0}=1, \\
(a)_{n}:=(a ; q)_{n}=\prod_{i=0}^{n-1}\left(1-a q^{i}\right),
\end{gathered}
$$

and

$$
(a)_{\infty}:=(a ; q)_{\infty}=\prod_{n=0}^{\infty}\left(1-a q^{n}\right)
$$

In Chap. 16 of his Second Notebook [12, p. 197; 3, p. 34], S. Ramanujan developed the theory of theta functions and his theta function is defined as follows:

$$
f(a, b):=\sum_{n=-\infty}^{\infty} a^{\frac{n(n+1)}{2}} b^{\frac{n(n-1)}{2}}=(-a ; a b)_{\infty}(-b ; a b)_{\infty}(a b ; a b)_{\infty}, \quad|a b|<1
$$

Following Ramanujan [12, p. 197], we define

$$
\begin{gathered}
\phi(q):=f(q, q)=1+2 \sum_{k=1}^{\infty} q^{k^{2}}=\frac{(-q ;-q)_{\infty}}{(q ;-q)_{\infty}}, \\
\psi(q):=f\left(q, q^{3}\right)=\sum_{k=0}^{\infty} q^{\frac{k(k+1)}{2}}=\frac{\left(q^{2} ; q^{2}\right)_{\infty}}{\left(q ; q^{2}\right)_{\infty}}
\end{gathered}
$$

University of Mysore, Mysore, India.

Published in Ukrains'kyi Matematychnyi Zhurnal, Vol. 62, No. 12, pp. 1609-1619, December, 2010. Original article submitted October 27, 2009; revision submitted July 8, 2010. 
and

$$
f(-q):=f\left(-q,-q^{2}\right)=\sum_{k=-\infty}^{\infty}(-1)^{k} q^{\frac{k(3 k-1)}{2}}=(q ; q)_{\infty} .
$$

For the sake of convenience, we denote

$$
f\left(-q^{n}\right)=f_{n} .
$$

The celebrated Rogers-Ramanujan continued fraction is defined as

$$
R(q):=\frac{q^{1 / 5} f\left(-q,-q^{4}\right)}{f\left(-q^{2},-q^{3}\right)}=\frac{q^{1 / 5}}{1}+\frac{q}{1}+\frac{q^{2}}{1}+\frac{q^{3}}{1}+\ldots
$$

On p. 365 of his Lost Notebook [13], Ramanujan recorded five identities showing the relationships between $R(q)$ and five continued fractions $R(-q), R\left(q^{2}\right), R\left(q^{3}\right), R\left(q^{4}\right)$, and $R\left(q^{5}\right)$. He also recorded these identities at the scattered places of his Notebooks [12]. L. J. Rogers [14] established the modular equations relating $R(q)$ and $R\left(q^{n}\right)$ for $n=2,3,5$, and 11. The last of these equations cannot be found in Ramanujan's works. For the proof of these equations, we can refer the reader, e.g., to [4]. Recently K. R. Vasuki and S. R. Swamy [19] found the modular equation relating $R(q)$ with $R\left(q^{7}\right)$.

The Ramanujan's cubic continued fraction $G(q)$ is defined as follows:

$$
G(q):=\frac{q^{1 / 3} f\left(-q,-q^{5}\right)}{f\left(-q^{3},-q^{3}\right)}=\frac{q^{1 / 3}}{1}+\frac{q+q^{2}}{1}+\frac{q^{2}+q^{4}}{1}+\frac{q^{3}+q^{6}}{1}+\ldots
$$

The continued fraction (1.2) was first introduced by Ramanujan in his second letter to G. H. Hardy [10]. He also recorded the continued fraction (1.2) on p. 365 of his Lost Notebook [13] and claimed that there are many results for $G(q)$ similar to the results obtained for the famous Rogers-Ramanujan continued fraction (1.1).

Motivated by Ramanujan's claim, H. H. Chan [5], N. D. Baruah [1], K. R. Vasuki, and B. R. Srivatsa Kumar [18] established the modular relations between $G(q)$ and $G\left(q^{n}\right)$ for $n=2,3,5,7,11$ and 13 .

The Ramanujan Göllnitz-Gordon continued fraction [13, p. 44; 8; 9] is defined as follows:

$$
H(q):=\frac{q^{1 / 2} f\left(-q^{3},-q^{5}\right)}{f\left(-q,-q^{7}\right)}=\frac{q^{1 / 2}}{1}+\frac{q^{2}}{1+q^{3}}+\frac{q^{4}}{1+q^{5}}+\ldots
$$

Chan and S. S. Hang [6] and K. R. Vasuki and B. R. Srivatsa Kumar [17] established the relationships between $H(q)$ and $H\left(q^{n}\right)$ with $n=3,4,5,11$ by using the modular equations deduced by Ramanujan. Recently, B. Cho, J. K. Koo, and Y. K. Park [7] extended the results cited above for the continued fraction (1.3) to all odd prime $p$ by computing the affine models of modular curves $X(\Gamma)$ with $\Gamma=\Gamma_{1}(8) \cap \Gamma_{0}(16 p)$.

Motivated by the cited works on the continued fractions (1.1)-(1.3), in the present paper, we establish the relationship between the following continued fraction $U(q)$ and $U\left(q^{n}\right)$ for $n=2,7,9,11$ and 13 :

$$
U(q):=\frac{q f\left(-q,-q^{11}\right)}{f\left(-q^{5},-q^{7}\right)} \frac{q(1-q)}{\left(1-q^{3}\right)}+\frac{q^{3}\left(1-q^{2}\right)\left(1-q^{4}\right)}{\left(1-q^{3}\right)\left(1+q^{6}\right)}+\frac{q^{3}\left(1-q^{8}\right)\left(1-q^{10}\right)}{\left(1-q^{3}\right)\left(1+q^{12}\right)}+\ldots
$$

The continued fraction (1.4) was established by M. S. M. Naika, et al. [11] as a special case of a fascinating continued-fraction identity recorded by Ramanujan in his Second Notebook [12, p. 74]. Furthermore, they have also established a modular relationship between the continued fraction $U(q)$ and $U\left(q^{n}\right)$ with $n=3$ and 5 . 


\section{Some Preliminary Results}

Theorem 2.1 [11]. We have

$$
\frac{\phi(q)}{\phi\left(q^{3}\right)}=\frac{1+U(q)}{1-U(q)}
$$

Theorem 2.2. We have

$$
\frac{\phi^{2}(-q)}{\phi^{2}\left(-q^{3}\right)}=1-\frac{4 U(q)}{1+U^{2}(q)}
$$

Proof. It follows from [2] that

$$
\left(\phi^{2}(q)+\phi^{2}\left(q^{3}\right)\right)^{2}=\frac{4 \phi\left(q^{3}\right) \phi^{3}\left(-q^{3}\right) \phi(q)}{\phi(-q)} .
$$

Moreover, it follows from [16] that

$$
\left(3 \phi^{2}\left(q^{3}\right)-\phi^{2}(q)\right)^{2}=\frac{4 \phi\left(q^{3}\right) \phi(q) \phi^{3}(-q)}{\phi\left(-q^{3}\right)} .
$$

From (2.3) and (2.4), we deduce the relation

$$
\frac{\phi^{2}(-q)}{\phi^{2}\left(-q^{3}\right)}=\frac{3-\frac{\phi^{2}(q)}{\phi\left(q^{3}\right)}}{1+\frac{\phi^{2}(q)}{\phi^{2}\left(q^{3}\right)}}
$$

By using (2.1) on the right-hand side of this identity, we find (2.2).

Theorem 2.3. We have

$$
\frac{\psi^{2}\left(q^{2}\right)}{q \psi^{2}\left(q^{6}\right)}=U(q)+\frac{1}{U(q)}-1
$$

Proof. From [2], we conclude that

$$
1-\frac{\phi^{2}(-q)}{\phi^{2}\left(-q^{3}\right)}=\frac{4 q f_{1} f_{12}^{3}}{f_{4} f_{3}^{3}}
$$

and, in addition, that

$$
1+\frac{\psi^{2}\left(q^{2}\right)}{q \psi^{2}\left(q^{6}\right)}=\frac{f_{3}^{3} f_{4}}{q f_{1} f_{12}^{3}} .
$$

By using (2.6) and the identity presented above, we find 


$$
1+\frac{\psi^{2}\left(q^{2}\right)}{q \psi^{2}\left(q^{6}\right)}=\frac{4}{1-\frac{\phi^{2}(-q)}{\phi^{2}\left(-q^{3}\right)}}
$$

In view of (2.2), this gives the required result.

Theorem 2.4. We have

$$
\frac{\psi^{2}(q)}{q^{1 / 2} \psi^{2}\left(q^{3}\right)}=\frac{1+U(q)}{1-U(q)} \sqrt{U(q)+\frac{1}{U(q)}-1 .}
$$

Proof. Consider the equality

$$
\frac{\psi^{4}(q)}{q \psi^{4}\left(q^{3}\right)}=\frac{\phi^{2}(q) \psi^{2}\left(q^{2}\right)}{q \phi^{2}\left(q^{3}\right) \psi^{2}\left(q^{6}\right)},
$$

where we have used the identity $\phi(q) \psi\left(q^{2}\right)=\psi^{2}(q)$ from Entry 25 [3, p. 40]. Further, by using (2.1) and (2.7) on the right-hand side of this equality, we obtain the required result.

Theorem 2.5. We have

$$
\frac{\psi^{4}(-q)}{q \psi^{4}\left(-q^{3}\right)}=\left[\frac{U^{2}(q)+1}{U(q)}+\frac{4 U(q)}{1+U^{2}(q)}-5\right]
$$

Proof. Changing $q$ into $-q$ in relation (2.8) and then using (2.2) and (2.5) on the right-hand side of the same relation, we obtain (2.9).

To prove our main results, we require the following easily deducible identities:

$$
\begin{gathered}
\phi(q)=\frac{f_{2}^{5}}{f_{1}^{2} f_{4}^{2}}, \quad \psi(q)=\frac{f_{2}^{2}}{f_{1}}, \\
\phi(-q)=\frac{f_{1}^{2}}{f_{2}}, \quad \psi(-q)=\frac{f_{1} f_{4}}{f_{2}}, \\
f(q)=\frac{f_{2}^{3}}{f_{1} f_{4}}, \quad \chi(q)=\frac{f_{2}^{2}}{f_{1} f_{4}}
\end{gathered}
$$

and

$$
\chi(-q)=\frac{f_{1}}{f_{2}}
$$

Moreover, we need the following identities analogous to the Rogers-Ramanujan forty identities:

Theorem 2.6 [15]. Let

$$
M(q)=\frac{f\left(-q^{5},-q^{7}\right)}{f_{4}}
$$


and

$$
N(q)=\frac{f\left(-q,-q^{11}\right)}{f_{4}}
$$

Then

$$
\begin{gathered}
M\left(q^{2}\right) N(q)+q M(q) N\left(q^{2}\right)=\frac{f_{3} f_{24}}{f_{4} f_{8}}, \\
M\left(q^{2}\right) N(q)-q M(q) N\left(q^{2}\right)=\frac{f_{1}^{2} f_{6} f_{24}}{f_{2} f_{3} f_{4} f_{8}}, \\
M\left(q^{3}\right) N(q)+q^{2} M(q) N\left(q^{3}\right)=\frac{f_{1} f_{6}^{5} f_{9} f_{36}}{f_{2}^{2} f_{3}^{2} f_{12}^{3} f_{18}}, \\
M\left(q^{3}\right) N(q)-q^{2} M(q) N\left(q^{3}\right)=\frac{f_{1} f_{18}^{2}}{f_{4} f_{9} f_{12}}, \\
M\left(q^{5}\right) N(q)-q^{4} M(q) N\left(q^{5}\right)=\frac{f_{1} f_{6}^{2} f_{30}}{f_{2} f_{3} f_{12} f_{20}},
\end{gathered}
$$

and

$$
M(q) M\left(q^{5}\right)-q^{6} N(q) N\left(q^{5}\right)=\frac{f_{5} f_{6} f_{30}^{2}}{f_{4} f_{10} f_{15} f_{60}} .
$$

A proof of identities (2.11)-(2.16) can be found in [15].

\section{Main Results}

In the present section, we deduce the relationship between the continued fraction $U(q)$ and $U\left(q^{n}\right)$ for $n=$ $2,3,5,7,9,11$, and 13 .

Theorem 3.1. Let $u:=U(q)$ and $v:=U\left(q^{2}\right)$. Then

$$
u^{2}-v+2 u v-u^{2} v+v^{2}=0 .
$$

Proof. By (1.4), we have

$$
U(q)=\frac{q N(q)}{M(q)} .
$$

Dividing (2.11) by (2.12) and using (2.10), we get

$$
\frac{M\left(q^{2}\right) N(q)+q M(q) N\left(q^{2}\right)}{M\left(q^{2}\right) N(q)-q M(q) N\left(q^{2}\right)}=\frac{\varphi\left(-q^{3}\right)}{\varphi(-q)} .
$$


Squaring both sides of this identity, using (3.1) on its left-hand side and (2.2) on its right-hand side, and then dividing the equality obtained as a result by $4 u$, we arrive at the required relation.

Theorem 3.2 [11]. Let $u=U(q)$ and $v=U\left(q^{3}\right)$. Then

$$
u^{3}-v^{3}+v^{2}-v+3 u v-3 u^{2} v^{2}+u^{3} v^{2}-u^{3} v=0
$$

Proof. Dividing (2.14) by (2.13) and using (2.10), we find

$$
\frac{M\left(q^{3}\right) N(q)-q^{2} M(q) N\left(q^{3}\right)}{M\left(q^{3}\right) N(q)+q^{2} M(q) N\left(q^{3}\right)}=\frac{\phi\left(-q^{2}\right) \phi\left(-q^{3}\right) \phi\left(-q^{18}\right)}{\phi\left(-q^{6}\right) \phi\left(-q^{9}\right) \phi\left(-q^{6}\right)}
$$

Squaring both sides of this identity and using relation (3.1) on its left-hand side and the identity

$$
\phi(q) \phi(-q)=\phi^{2}\left(-q^{2}\right)
$$

on its right-hand side, we obtain

$$
\left(\frac{U(q)-U\left(q^{3}\right)}{U(q)+U\left(q^{3}\right)}\right)^{2}=\frac{\phi(q)}{\phi\left(q^{3}\right)} \frac{\phi(-q)}{\phi\left(-q^{3}\right)} \frac{\phi\left(-q^{3}\right)}{\phi\left(-q^{9}\right)} \frac{\phi\left(q^{9}\right)}{\phi\left(q^{3}\right)} .
$$

Squaring again both sides of this identity, applying (2.1) and (2.2) on its right-hand side, and then factorizing by using Maple, we conclude that

$$
\begin{aligned}
8\left(-v^{3}\right. & \left.+v^{2}-v+3 u v-3 u^{2} v^{2}+u^{3} v^{2}-u^{3} v+u^{3}\right) \\
& \times\left(u^{5} v-u^{4} v^{3}+u^{4} v^{2}-3 u^{4} v+5 u^{3} v^{3}-u^{3} v^{2}-u^{3} v+u^{3}-u^{2} v^{5}+u^{4} v^{2}\right. \\
& \left.+u^{2} v^{3}-5 u^{2} v^{2}+3 v^{4} u-u v^{3}+u v^{2}-v^{4}\right)=0 .
\end{aligned}
$$

Thus, it follows from the definition of $u$ and $v$ that

$$
u=U(q)=1-q+q^{5}-q^{6}+q^{7}+\ldots
$$

and

$$
v=U\left(q^{3}\right)=1-q^{3}+q^{15}-q^{18}+q^{21}+\ldots
$$

By using (3.2) and the last relation in the same factors as above, we see that the first factor becomes

$$
q^{3}\left(3-6 q+3 q^{2}-3 q^{3}+6 q^{4}+3 q^{5}-9 q^{6}+\ldots\right)
$$

and the second factor becomes

$$
-q^{3}\left(10-22 q+15 q^{2}-22 q^{3}+33 q^{4}-9 q^{5}+\ldots\right) .
$$


Thus, the second factor does not vanish. Hence, by the identity Theorem, we must have

$$
v^{3}-v^{2}+v-3 u v+3 u^{2} v^{2}-u^{3} v^{2}+u^{3} v-u^{3}=0
$$

Theorem 3.2 is proved.

Theorem 3.3 [11]. Let $u:=U(q)$ and $v:=U\left(q^{5}\right)$. Then

$$
\begin{aligned}
& u v^{6}-u^{6} v^{5}+5 u^{5} v^{5}-5 u^{4} v^{5}-5 u v^{5}+10 v^{4} u^{3}+5 u v^{4} \\
& \quad-10 u^{4} v^{3}-10 u^{2} v^{3}+5 u^{5} v^{2}+10 u^{3} v^{2}-5 u^{5} v-5 u^{2} v+5 u v-v+u^{5}=0 .
\end{aligned}
$$

Proof. Dividing (2.15) by (2.16) and using (2.10), we find

$$
\frac{M\left(q^{5}\right) N(q)-q^{4} M(q) N\left(q^{5}\right)}{M(q) M\left(q^{5}\right)-q^{6} N(q) N\left(q^{5}\right)}=\frac{q \psi(-q)}{\psi\left(-q^{3}\right)} \frac{\psi\left(-q^{15}\right)}{\psi\left(-q^{5}\right)} .
$$

Taking power 4 of both sides of this identity, employing relation (2.9) on its right-hand side, and then factorizing by using Maple, we conclude that

$$
\begin{aligned}
& \left(u^{2}+u^{2} v^{2}+1-4 u v+v^{2}\right)\left(u v^{6}-u^{6} v^{5}+5 u^{5} v^{5}-5 u^{4} v^{5}-5 u v^{5}+10 v^{4} u^{3}\right. \\
& \left.\quad+5 u v^{4}-10 u^{4} v^{3}-10 u^{2} v^{3}+5 u^{5} v^{2}+10 u^{3} v^{2}-5 u^{5} v-5 u^{2} v+5 u v-v+u^{5}\right)=0 .
\end{aligned}
$$

Changing $q$ into $q^{5}$ in (3.2), we obtain

$$
v=U\left(q^{5}\right)=1-q^{5}+q^{25}-q^{30}+q^{35}+\ldots
$$

By using (3.2) and the last relation in the same factors as above, we see that the first factor becomes

$$
q^{2}\left(2-4 q^{4}+2 q^{5}-4 q^{6}+4 q^{8}-2 q^{9}+3 q^{10}+\ldots\right)
$$

and the second factor becomes

$$
-q^{7}\left(40-40 q+20 q^{2}+10 q^{3}-55 q^{4}+110 q^{5}-180 q^{6}+\ldots\right) .
$$

Thus, the first factor does not vanish. Hence, by the identity theorem, we must have

$$
\begin{aligned}
& u v^{6}-u^{6} v^{5}+5 u^{5} v^{5}-5 u^{4} v^{5}-5 u v^{5}+10 v^{4} u^{3}+5 u v^{4}-10 u^{4} v^{3} \\
& \quad-10 u^{2} v^{3}+5 u^{5} v^{2}+10 u^{3} v^{2}-5 u^{5} v-5 u^{2} v+5 u v-v+u^{5}=0 .
\end{aligned}
$$

Theorem 3.3 is proved. 
Theorem 3.4. Let $u=U(q)$ and $v=U\left(q^{7}\right)$. Then

$$
\begin{aligned}
& 7 u^{3} v^{2}-35 u^{3} v^{4}-7 u^{3} v-7 u^{3} v^{3}+35 u^{4} v^{3}-u^{7} \\
& +7 u^{5} v-7 u^{6} v+7 u^{7} v-28 u^{5} v^{2}+28 u^{6} v^{2}-14 v^{2} u^{7}+7 v^{3} u^{5}-7 v^{3} u^{6} \\
& +7 v^{3} u^{7}-35 v^{4} u^{5}+7 v^{5} u-7 v^{5} u^{2}+7 v^{5} u^{3}+35 v^{5} u^{4}-7 v^{5} u^{5}+28 v^{5} u^{6} \\
& -7 v^{5} u^{7}-14 v^{6} u+28 v^{6} u^{2}-28 v^{6} u^{3}+7 v^{6} u^{5}-28 v^{6} u^{6}+7 v^{6} u^{7}+7 v^{7} u \\
& -7 v^{7} u^{2}+7 v^{7} u^{3}-7 v^{7} u^{5}+14 v^{7} u^{6}-7 v^{7} u^{7}+v^{7} u^{8}-v^{8} u+v-7 u v \\
& +7 u v^{2}+14 u^{2} v-28 u^{2} v^{2}-7 u v^{3}+28 u^{2} v^{3}=0 .
\end{aligned}
$$

Proof. If

$$
P=\frac{\phi(q)}{\phi\left(q^{3}\right)}
$$

and

$$
Q=\frac{\phi\left(q^{7}\right)}{\phi\left(q^{21}\right)}
$$

then

$$
\begin{aligned}
\left(\frac{Q}{P}\right)^{4}- & \left(\frac{P}{Q}\right)^{4}+14\left[\left(\frac{Q}{P}\right)^{2}-\left(\frac{P}{Q}\right)^{2}\right] \\
& =(P Q)^{3}+\frac{27}{(P Q)^{3}}+7\left(P Q+\frac{3}{P Q}\right)\left[1-\left(\frac{P}{Q}\right)^{2}-\left(\frac{Q}{P}\right)^{2}\right]
\end{aligned}
$$

We have deduced identity (3.3) on the lines remarked by N. D. Baruah [1]. Thus, by using (2.1) in (3.3), we arrive at the required result.

Theorem 3.5. Let $u=U(q)$ and $v=U\left(q^{9}\right)$. Then

$$
\begin{aligned}
9 u^{4} v & +126 v^{4} u-90 u v^{3}+27 v^{8} u^{3}+v-9 v u+45 v^{2} u+378 u^{7} v^{5} \\
& +135 u^{8} v^{4}+54 u^{8} v^{2}-9 u^{8} v+18 u^{7} v+558 v^{4} u^{6}-558 v^{5} u^{3}+561 v^{4} u^{3} \\
& -10 u^{9} v^{2}-333 u^{6} v^{3}+4 u^{9} v-561 u^{6} v^{5}+16 u^{9} v^{3}-19 u^{9} v^{4}+369 v^{5} u^{2} \\
& -99 u^{5} v^{2}-378 v^{4} u^{2}+135 v^{7} u^{2}+99 u v^{6}+16 u^{9} v^{5}+333 u^{3} v^{6}
\end{aligned}
$$




$$
\begin{aligned}
& +243 u^{7} v^{3}-243 u^{2} v^{6}-126 u^{8} v^{5}-27 u^{6} v-54 v^{7} u+9 v^{8} u-135 u^{7} v^{2} \\
& +10 v^{3}-198 v^{6} u^{4}+369 u^{4} v^{5}-16 v^{6}+19 v^{5}-16 v^{4}-u^{9}-4 v^{8} \\
& +10 v^{7}-243 v^{4} u^{4}-153 v^{2} u^{2}+99 v^{7} u^{4}+297 v^{3} u^{2}+9 u^{8} v^{8}+243 u^{5} v^{5} \\
& -18 v^{8} u^{2}-297 v^{6} u^{7}+30 u^{6} v^{8}+153 v^{7} u^{7}-30 v u^{3}-165 v^{7} u^{3} \\
& +198 u^{5} v^{3}-99 u^{8} v^{3}-423 v^{3} u^{3}-9 u^{4} v^{8}-369 u^{7} v^{4}+165 u^{6} v^{2} \\
& +423 u^{6} v^{6}-153 u^{5} v^{6}-171 u^{6} v^{7}-369 u^{5} v^{4}-9 u^{5} v^{8}+54 u^{5} v^{7} \\
& +27 v u^{2}+90 u^{8} v^{6}+v^{9}+9 u^{5} v-10 u^{9} v^{6}-45 u^{8} v^{7}+171 v^{2} u^{3} \\
& +153 v^{3} u^{4}+4 u^{9} v^{7}-4 v^{2}-27 u^{7} v^{8}-54 u^{4} v^{2}-u^{9} v^{8}-135 v^{5} u=0 .
\end{aligned}
$$

Proof. Let $w=U\left(q^{3}\right)$. Then, by Theorem 3.2, we get

$$
u^{3}-w^{3}+w^{2}-w+3 u w-3 u^{2} w^{2}+u^{3} w^{2}-u^{3} w=0 .
$$

Changing $q$ into $q^{3}$ in relation (3.4), we obtain

$$
w^{3}-v^{3}+v^{2}-v+3 w v-3 w^{2} v^{2}+w^{3} v^{2}-w^{3} v=0
$$

Further, eliminating $w$ between (3.4) and (3.5), we deduce the required result.

Theorem 3.6. Let $u=U(q)$ and $v=U\left(q^{11}\right)$. Then

$$
\begin{aligned}
-363 v^{7} u^{10}- & 528 v^{10} u^{8}+11 v^{10} u^{11}-1089 v^{9} u^{8}+1463 v^{4} u^{8}-1012 v^{7} u^{8} \\
& +11 v^{11} u^{2}+v u-968 v^{4} u^{4}+44 v^{3} u-374 v^{3} u^{2}+77 v^{4} u \\
& -528 v^{4} u^{2}-11 v u^{2}-363 v^{5} u^{2}+55 v^{7} u^{11}+v^{11} u^{11} \\
& +759 v^{7} u^{9}+55 v^{5} u+55 v^{1} 1 u^{7}-759 v^{3} u^{7}-55 v^{7} u \\
& +363 v^{7} u^{2}-77 v^{8} u+528 v^{8} u^{2}+176 v^{10} u^{2} \\
& +44 v^{11} u^{9}+363 v^{2} u^{5}-44 v u^{9}-11 v^{2} u^{11}-11 v^{10} u \\
& +528 v^{10} u^{4}+528 v^{4} u^{10}+374 v^{9} u^{2}-44 v^{9} u
\end{aligned}
$$




$$
\begin{aligned}
& -110 v^{2} u^{2}+11 v^{2} u+77 v u^{8}+374 v^{3} u^{10}-363 v^{2} u^{7} \\
& -77 v u^{4}+11 v u^{10}+1089 v^{4} u^{3}-374 v^{9} u^{10} \\
& +44 v u^{3}-759 v^{9} u^{5}+55 v u^{5}+759 v^{5} u^{3} \\
& +363 v^{5} u^{10}+1012 v^{5} u^{8}-1012 v^{5} u^{4}-55 v u^{7}+1496 v^{5} u^{5} \\
& -759 v^{5} u^{9}-528 v^{2} u^{4}-704 v^{5} u^{7}-55 v^{5} u^{11}-968 v^{8} u^{8} \\
& +1463 v^{8} u^{4}+1089 v^{3} u^{8}-528 v^{8} u^{10}-1089 v^{3} u^{4} \\
& -1089 v^{8} u^{3}-1012 v^{8} u^{5}+1496 v^{7} u^{7}+77 v^{8} u^{11} \\
& +1089 v^{8} u^{9}-363 v^{10} u^{5}+1012 v^{8} u^{7}+u^{12}-374 v^{2} u^{9} \\
& +1023 v^{3} u^{3}-110 v^{10} u^{10} 1012 v^{7} u^{4}-704 v^{7} u^{5}-77 v^{11} u^{8} \\
& +1012 v^{4} u^{5}+11 v u^{11}-44 v^{11} u^{3}-1012 v^{4} u^{7}+924 v^{6} u^{6} \\
& +1089 v^{9} u^{4}+1023 v^{9} u^{9}-759 v^{7} u^{3}+759 v^{3} u^{5}+44 v^{9} u^{11} \\
& +528 v^{2} u^{8}+759 v^{9} u^{7}-803 v^{3} u^{9}-44 v^{3} u^{11}+363 v^{10} u^{7} \\
& +1089 v^{4} u^{9}+11 v^{11} u+374 v^{2} u^{3}-77 v^{4} u^{11}+77 v^{11} u^{4} \\
& +374 v^{10} u^{9}-55 v^{11} u^{5}+v^{12}-374 v^{10} u^{3}-11 v^{11} u^{10}-803 v^{9} u^{3}+176 v^{2} u^{10}=0 .
\end{aligned}
$$

Proof. Let

$$
P=\frac{\varphi(q)}{\varphi\left(q^{3}\right)}
$$

and

$$
Q=\frac{\varphi\left(q^{11}\right)}{\varphi\left(q^{33}\right)}
$$

Then it follows from [18] that 


$$
\begin{aligned}
&(P Q)^{5}+\frac{3^{5}}{(P Q)^{5}}- 11\left[(P Q)^{3}+\frac{3^{3}}{(P Q)^{3}}\right]+308\left[P Q+\frac{3}{P Q}\right] \\
&=\left(\frac{P}{Q}\right)^{6}+\left(\frac{Q}{P}\right)^{6}+22\left[\left(\frac{P}{Q}\right)^{4}+\left(\frac{Q}{P}\right)^{4}\right]\left[3-\left(P Q+\frac{3}{P Q}\right)\right] \\
&+11\left[\left(\frac{P}{Q}\right)^{2}+\left(\frac{Q}{P}\right)^{2}\right]\left[(P Q)^{3}+\frac{3^{3}}{(P Q)^{3}}-15\left(P Q+\frac{3}{P Q}\right)+45\right]+924
\end{aligned}
$$

By using (2.1) in the last relation, we arrive at the required result.

Theorem 3.7. Let $u=U(q)$ and $v=U\left(q^{13}\right)$. Then

$$
\begin{aligned}
-8346 u^{8} v^{5} & +611 u^{11} v^{12}+6318 u^{4} v^{8}+8723 u^{8} v^{6}-8346 u^{5} v^{8}-78 u^{7} v^{5} \\
& +910 u^{10} v^{7}+1508 u^{8} v^{2}-780 u^{8} v^{7}+8476 u^{8} v^{9}-10322 u^{4} v^{9}-5148 u^{8} v^{8} \\
& -481 u^{3} v^{12}-7228 u^{8} v^{10}+10894 u^{5} v^{9}+611 u^{3} v^{2}-208 u^{6} v^{13}+910 u^{7} v^{10} \\
& -10322 u^{5} v^{10}-143 u^{12} v^{12}+6409 u^{3} v^{9}-1911 u^{5} v^{12}+1313 u^{10} v^{2} \\
& -832 u^{7} v^{11}-5317 u^{3} v^{10}-130 u^{7} v^{12}-2184 u^{11} v^{11}-182 u^{13} v^{9}+2652 u^{3} v^{11} \\
& -5148 u^{6} v^{6}-5317 u^{10} v^{3}-91 u^{13} v^{4}+1508 u^{6} v^{12}+78 u v^{7}+8723 u^{6} v^{8} \\
& +4706 u^{6} v^{3}+1846 u^{5} v^{2}+1508 u^{2} v^{8}+v^{14}+9607 u^{10} v^{4}-5317 u^{4} v^{11} \\
& -182 u v^{5}+78 u^{6} v-1170 u^{6} v^{2}-2184 u^{3} v^{3}-7228 u^{4} v^{6}-6331 u^{11} v^{9} \\
& +195 u^{9} v+10894 u^{9} v^{5}+6409 u^{9} v^{3}-7228 u^{10} v^{8}+6318 u^{10} v^{6}+78 u^{7} v^{13} \\
& +910 u^{7} v^{4}-130 u^{7} v^{2}+78 u^{7} v+10257 u^{4} v^{5}+u^{14}-832 u^{3} v^{7}-78 u^{7} v^{9} \\
& +4706 u^{3} v^{6}-780 u^{6} v^{7}+13 u^{13} v^{12}-130 u^{2} v^{7}+52 u^{2} v^{13}-1911 u^{9} v^{2} \\
& +6318 u^{8} v^{4}+78 u v^{6}+4407 v^{11} v^{10}+1846 u^{2} v^{5}-8346 u^{6} v^{9}-1768 u^{7} v^{7} \\
& -8346 u^{9} v^{6}-832 u^{7} v^{3}-10322 u^{10} v^{5}-7631 u^{4} v^{4}-3926 u^{8} v^{3}-780 u^{7} v^{8} \\
& \\
& \\
& \\
&
\end{aligned}
$$




$$
\begin{aligned}
& +846 u^{12} v^{9}-u^{13} v^{13}+156 u^{13} v^{10}-10322 u^{9} v^{4}+4407 u^{4} v^{3}+13 u v^{2} \\
& +10257 u^{9} v^{10}+6409 u^{11} v^{5}-6331 u^{5} v^{3}-1378 u^{4} v^{2}+13 u^{2} v+4407 u^{10} v^{11} \\
& -3926 u^{11} v^{6}-3926 u^{3} v^{8}-143 u^{2} v^{2}-1911 u^{12} v^{5}+6409 u^{5} v^{11}+8476 u^{6} v^{5} \\
& +156 u v^{4}-65 u^{3} v+1508 u^{12} v^{6}-208 u v^{8}+13 u^{12} v^{13}-65 u^{11} v^{13}+156 u^{10} v^{13} \\
& +195 u^{13} v^{5}-1378 u^{10} v^{12}+1313 u^{4} v^{12}-91 u^{4} v^{13}-130 u^{12} v^{7}-3926 u^{6} v^{11} \\
& -832 u^{11} v^{7}-65 u v^{3}-208 u^{13} v^{6}-1170 u^{12} v^{8}+9607 u^{4} v^{10}-13 u^{3} v^{13} \\
& +6318 u^{6} v^{10}+78 u^{13} v^{8}+611 u^{12} v^{11}+156 u^{4} v+611 u^{2} v^{3}+78 u^{13} v^{7} \\
& +4706 u^{11} v^{8}-1378 u^{2} v^{4}+10257 u^{5} v^{4}-208 u^{8} v-26 u^{13} v-7228 u^{6} v^{4} \\
& -6331 u^{3} v^{5}-5317 u^{11} v^{4}+1313 u^{12} v^{4}-12909 u^{5} v^{5}-78 u^{5} v^{7}=0 . \\
& -91 u^{10} v+52 u^{12} v+4407 u^{3} v^{4}-13 u^{11} v+8476 u^{5} v^{6}-481 u^{11} v^{2}-1378 u^{12} v^{10} \\
& -1170 u^{8} v^{12}+78 u^{8} v^{13}+4706 u^{8} v^{11}-182 u^{5} v-13 u v^{11}+195 u v^{9}-91 u v^{10} \\
& -65 u^{13} v^{11}-u v-39 u^{2} v^{12}-481 u^{2} v^{11}-26 u v^{13}-1170 u^{2} v^{6}+52 u v^{12} \\
& -1846 u^{9} v^{12}+910 u^{4} v^{7}-6331 u^{9} v^{11}-13 u^{13} v^{3}+2652 u^{11} v^{3} \\
& -182 u^{9} v^{13}+52 u^{13} v^{2}+195 u^{5} v^{13}+1313 u^{2} v^{10}-39 u^{12} v^{2} \\
& +30
\end{aligned}
$$

Proof. Let

$$
P=\frac{\varphi(q)}{\varphi\left(q^{3}\right)} \quad \text { and } \quad Q=\frac{\varphi\left(q^{13}\right)}{\varphi\left(q^{39}\right)}
$$

Then it follows from [18] that

$$
\begin{aligned}
\left(\frac{Q}{P}\right)^{7}+\left(\frac{P}{Q}\right)^{7} & +13\left[\left(\frac{P}{Q}\right)^{6}+\left(\frac{Q}{P}\right)^{6}\right]-26\left[\left(\frac{P}{Q}\right)^{5}+\left(\frac{Q}{P}\right)^{5}\right] \\
& -13\left[3(P Q)^{2}+\frac{27}{(P Q)^{2}}+10\right]\left[\left(\frac{Q}{P}\right)^{4}+\left(\frac{P}{Q}\right)^{4}\right]+13\left[10(P Q)^{2}+\frac{90}{(P Q)^{2}}+68\right]
\end{aligned}
$$




$$
\begin{aligned}
& \times\left[\left(\frac{Q}{P}\right)^{3}+\left(\frac{P}{Q}\right)^{3}\right]+13\left[10(P Q)^{2}+\frac{90}{(P Q)^{2}}+68\right]\left[\left(\frac{Q}{P}\right)^{3}+\left(\frac{P}{Q}\right)^{3}\right] \\
& +13\left[(P Q)^{4}+\frac{81}{(P Q)^{4}}-20\left((P Q)^{2}+\frac{9}{(P Q)^{2}}\right)-115\right]\left[\left(\frac{Q}{P}\right)^{2}+\left(\frac{P}{Q}\right)^{2}\right] \\
& -13\left[(P Q)^{4}+\frac{81}{(P Q)^{4}}-10\left((P Q)^{2}+\frac{9}{(P Q)^{2}}\right)-131\right]\left[\left(\frac{Q}{P}\right)+\left(\frac{P}{Q}\right)\right] \\
& =(P Q)^{6}+\frac{729}{(P Q)^{6}}-26\left[(P Q)^{4}+\frac{81}{(P Q)^{4}}\right]+169\left[(P Q)^{2}+\frac{9}{(P Q)^{2}}\right]+832 .
\end{aligned}
$$

Finally, by using (2.1) in the last identity, we arrive at the required result.

\section{Acknowledgements}

The authors are thankful to the DST, New Delhi, for awarding the research project [No. SR/S4/MS:517/08] under which the present work has been done. Further, the authors thank the referee for the comments aimed at improving the manuscript.

\section{REFERENCES}

1. N. D. Baruah, "Modular equations for Ramanujan's cubic continued fraction,” J. Math. Anal. Appl., 268, 244-255 (2002).

2. N. D. Baruah and R. Barman, "Certain Theta-function identities and Ramanujan's modular equations of degree 3," Indian J. Math., 48, No. 1, 113-133 (2006).

3. B. C. Berndt, Ramanujan Notebooks. Pt. III, Springer, New York (1991).

4. B. C. Berndt, Ramanujan Notebooks. Pt. V, Springer, New York (1998).

5. H. H. Chan, "On Ramanujan's cubic continued fraction," Acta Arithm., 73, No. 4, 343-355 (1995).

6. H. H. Chan and S. S. Huang, "On the Ramanujan-Göllnitz-Gordan continued fraction,” Ramanujan J., 1., 75-90 (1997).

7. B. Cho, J. K. Koo, and Y. K. Park, “Arithmetic of the Ramanujan-Göllnitz-Gordan continued fraction,” J. Number Theory, 4, No. 129, 922-947 (2009).

8. H. Göllnitz, "Partition mit Differenzebedingungen," J. Reine Angew. Math., 25, 154-190 (1967).

9. B. Gordon, "Some continued fractions of the Rogers-Ramanujan type," Duke Math. J., 32, 741-748 (1965).

10. G. H. Hardy, Ramanujan, Chelsea, New York (1978).

11. M. S. M. Naika, B. N. Dharmendra, and K. Shivashankra, "A continued fraction of order twelve," Centr. Eur. J. Math., DOI, 10.2478/s 11533-008-0031-y.

12. S. Ramanujan, Notebooks, Tata Inst. Fundam. Research, Bombay (1957).

13. S. Ramanujan, The "Lost" Notebook and Other Unpublished Papers, Narosa, New Delhi (1988).

14. L. J. Rogers, "On a type of modular relation," Proc. London Math. Soc., 19, 387-397 (1920).

15. K. R. Vasuki and P. S. Guruprasad, "On certain new modular relations for the Rogers-Ramanujan type functions of order twelve," Proc. Jangjeon Math. Soc. (to appear).

16. K. R. Vasuki, G. Sharath, and K. R. Rajanna, "Two modular equations for squares of the cubic functions with applications," Note Math. (to appear).

17. K. R. Vasuki and B. R. Srivatsa Kumar, "Certain identities for Ramanujan-Göllnitz-Gordan continued fraction," J. Comput. Appl. Math., 187, 87-95 (2006).

18. K. R. Vasuki and B. R. Srivatsa Kumar, Two Identities for Ramanujan's Cubic Continued Fraction, Preprint.

19. K. R. Vasuki and S. R. Swamy, "A new identity for the Rogers-Ramanujan continued fraction,” J. Appl. Math. Anal. Appl., 2, No. 1, 71-83 (2006). 